$=$ Supporting Information $=$

\title{
Dioxygenation of Human Serum Albumin Having a Prosthetic Heme Group in a Tailor-Made Heme Pocket
}

Teruyuki Komatsu, ${ }^{\dagger, *}$ Naomi Ohmichi, ${ }^{\dagger}$ Patricia A. Zunszain, ${ }^{\ddagger}$ Stephen Curry, ${ }^{\ddagger}$ and Eishun Tsuchida ${ }^{\dagger, *}$

${ }^{\dagger}$ Advanced Research Institute for Science and Engineering, Waseda University, 3-4-1 Okubo, Shinjuku-ku, Tokyo 169-8555, Japan.

*Department of Biological Sciences, Imperial College London, Huxley Building, South Kensington Campus, London SW7 2AZ, United Kingdom.

e-mail; eishun@waseda.jp 


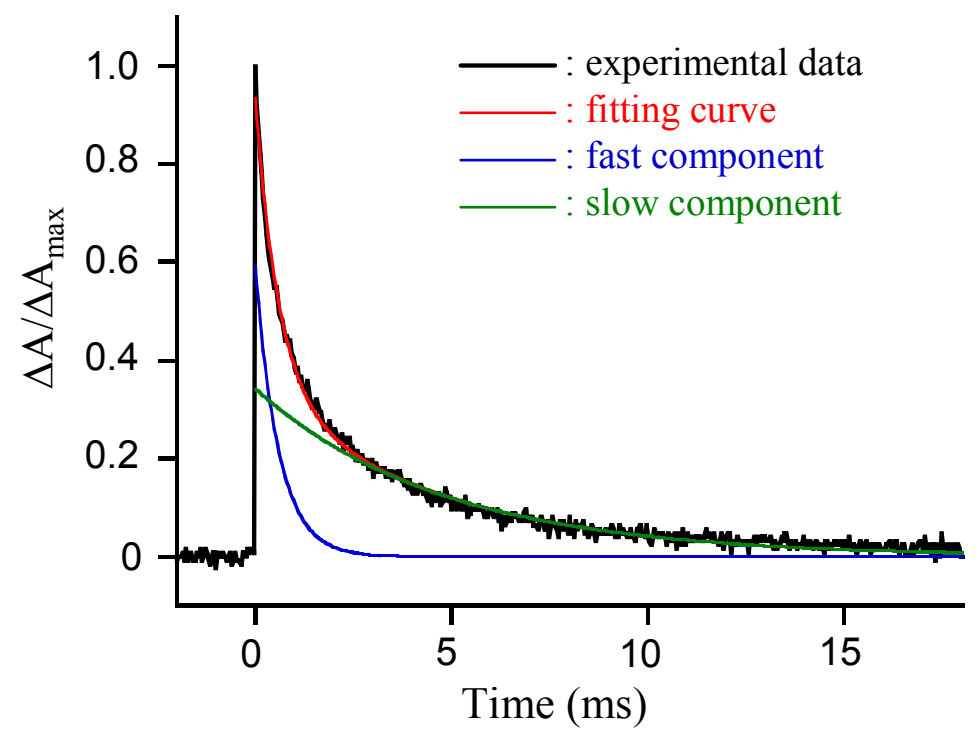

Fig. S1 Absorption decay of $\mathrm{CO}$ rebinding to rHSA(B)-heme after the laser flash photolysis at $22^{\circ} \mathrm{C}$. The kinetics was composed of two phases and relaxation curve was fitted by double-exponentials.

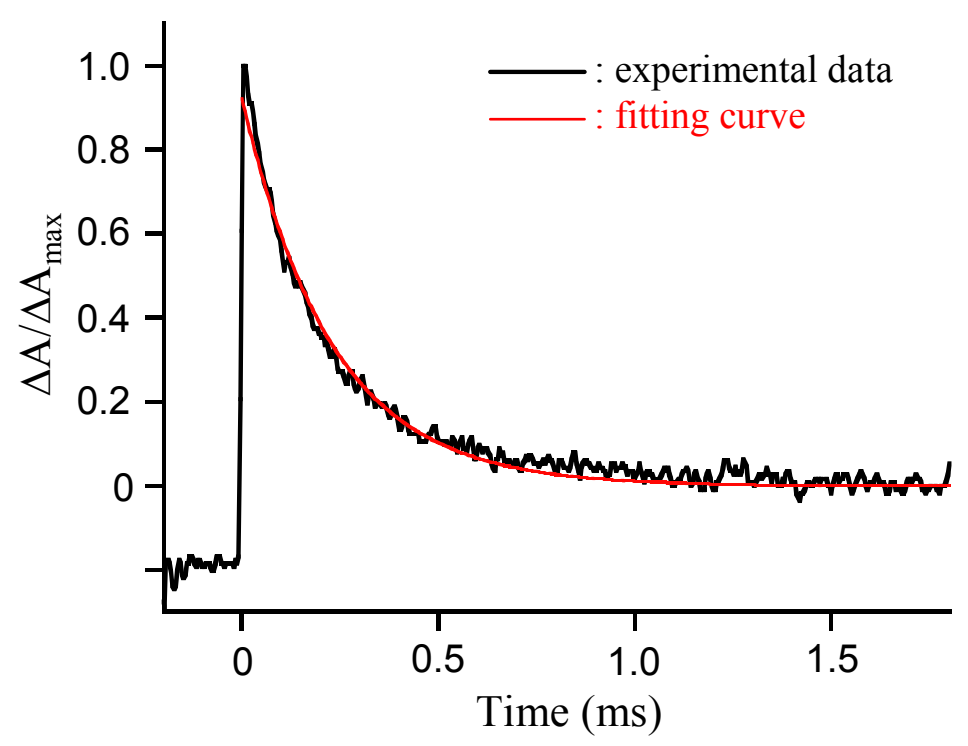

Fig. S2 Absorption decay of $\mathrm{O}_{2}$ rebinding to rHSA(B)-heme after the laser flash photolysis at $22^{\circ} \mathrm{C}$. The kinetics was composed of mono phase and relaxation curve was fitted by singleexponential. 